\title{
Range-azimuth decouple beamforming for frequency diverse array with
} Costas-sequence modulated frequency offsets

\author{
Zhe Wang, Wen-Qin Wang * (D) and Huaizong Shao
}

\begin{abstract}
Different from the phased-array using the same carrier frequency for each transmit element, the frequency diverse array (FDA) uses a small frequency offset across the array elements to produce range-angle-dependent transmit beampattern. FDA radar provides new application capabilities and potentials due to its range-dependent transmit array beampattern, but the FDA using linearly increasing frequency offsets will produce a range and angle coupled transmit beampattern. In order to decouple the range-azimuth beampattern for FDA radar, this paper proposes a uniform linear array (ULA) FDA using Costas-sequence modulated frequency offsets to produce random-like energy distribution in the transmit beampattern and thumbtack transmit-receive beampattern. In doing so, the range and angle of targets can be unambiguously estimated through matched filtering and subspace decomposition algorithms in the receiver signal processor. Moreover, random-like energy distributed beampattern can also be utilized for low probability of intercept (LPI) radar applications. Numerical results show that the proposed scheme outperforms the standard FDA in focusing the transmit energy, especially in the range dimension.
\end{abstract}

Keywords: Frequency diverse array (FDA) radar, Frequency offset, Range-dependent, Decouple, Costas sequence

\section{Introduction}

Active phased-array has been widely adopted in many applications such as radar, electronic warfare, radio astronomy, etc., because it can steer the beam electronically with high effectiveness [1]. The offered directional gain is useful for detecting/tracking weak targets and suppressing sidelobe interferences in other directions [2]. Since phased-array has range-independent transmit beampattern, the range and angle of targets cannot be unambiguously estimated from the beamforming output peaks. If we want to steer the array beams to multiple different range cells, multiple antennas or a multibeam antenna will be required. More importantly, controlling range-dependent energy distribution becomes an increasingly important requirement in many applications. While techniques exist in mitigating range-dependent interferences, e.g., space-time

\footnotetext{
*Correspondence: wqwang@uestc.edu.cn

School of Communication and Information Engineering, University of Electronic Science and Technology of China, Xiyuan Road, 611731 Chengdu, China
}

adaptive processing [3], they generally require a high computational cost.

In order to overcome the disadvantage of phased-array radar, the frequency diverse array (FDA) radar using a small frequency offset across the array elements was proposed in 2006 [4]. This stepped-frequency offset results in that the beam can scan the space in a periodic manner $[5,6]$. Its beamforming focusing direction will change as a function of the range, angle, time, and even the frequency offset [7]. These characteristics contrast with the range-independent transmit beampattern in a phase-array radar. FDA radar is different from orthogonal frequency division multiplexing (OFDM) radar [8] and multiple-input multiple-output (MIMO) radar $[9,10]$. OFDM radar uses orthogonal subcarriers, but nonorthogonal carriers are employed in FDA radar. MIMO radar aims to provide non-coherent waveforms to obtain increased degrees of freedom (DOFs), whereas FDA radar transmits overlapping signals with closely spaced frequencies to provide additional functionality. FDA radar is also different from conventional frequency scanning radar using the frequency increments as a function of time 
for all the elements [11], but FDA frequency offsets are characterized by the element index [12]. Another similar concept is the time-modulation array [13], which weights each element using on/off switching operation. FDA was investigated in [14] as a range-dependent beam with applications in suppressing range ambiguous clutter. Secmen et al. [5] described the time and angle periodicity of FDA radiation pattern. Higgins and Blunt [15] explored rangeangle coupled beamforming in FDA. Additional studies to exploit FDA range-dependent beampattern characteristics were reported in $[16,17]$. In fact, with the introduction of frequency increment, the FDA apparent angle will be different from its nominal beam scanning angle.

Due to its promising application potentials [18], FDA has sparked many interesting investigations [19-21]. Since FDA offers a range-angle-dependent beampattern, it is of great importance as this provides a potential for range-angle localization of targets, but the transmit beampattern of a standard FDA using linearly increasing frequency offsets is coupled in the range-angle dimension. This limits its application for unambiguously estimating target parameters. To decouple the range-angle coupling response of targets, a simple range-azimuth localization of targets is proposed in [22] by adopting a uniform linear array (ULA) double-pulse FDA radar. This double-pulse FDA radar transmits two pulses with zero and non-zero frequency offsets, respectively. In [23], a subarray-based FDA is proposed for target range-angle estimation. Furthermore, a transmit subaperturing is designed in [20] with convex optimization, so that the range and angle responses are decoupled and the equivalent transmit beam can be focused in a certain range-angle sector to localize the targets.

In [19], a nonuniform linear array is adopted for the FDA. However, the transmitter and receiver must be placed accurately. Another nonuniform linear array for FDA is attempted to suppress/locate range-dependent interference/target in [12], but the carrier frequency and/or frequency increments cannot be altered in realtime because it requires relocating the elements mechanically. Logarithmically increasing frequency offsets [24] or time-dependent frequency offsets [25] are also suggested to decouple the range-angle beampattern, but they result in poor beamforming performance, especially in the range dimension. In fact, the best decoupling approach is to form a dot-shaped beampattern rather than an "S"-shaped beampattern. Such a dot-shaped range-angle beampattern is synthesized in [26] by a symmetrical FDA using multicarrier frequency offsets and convex optimization, and in [27] by the use of random frequency offsets. Nevertheless, these two schemes are very difficult to implement in practical array systems.

In this paper, we analyze the reason of FDA range and angle coupled transmit beampattern and thus propose the range-azimuth decouple beamforming for FDA radar using Costas-sequence modulated frequency offsets. The rest of this paper is organized as follows. Section 2 provides a brief introduction to basic FDA radar scheme and motivation of this paper. Then, Section 3 proposes the range-azimuth decoupling beamforming for the FDA using Costas-sequence modulated frequency offsets. Finally, numerical results are provided in Section 4 and concluding summaries are drawn in Section 5.

\section{Basic FDA radar and motivation}

In conventional phased-arrays, it is assumed that the same waveform is radiated by each array elements. Different from conventional phased-arrays, FDA elements can be excited by either the same waveform or different waveforms. Without loss of generality, we assume that the waveform radiated from each array element is identical with a frequency offset of $\Delta f \mathrm{~Hz}$, as shown in Fig. 1, where the $x$-axis and $x$-axis are defined as along the linear array arrangement and the zero-azimuth angle, respectively. Therefore, the monochromatic continuous signal transmitted by the $m$ th element is

$$
s_{m}(t)=w_{m} w_{e}\left(f_{m}\right) \exp \left(-j 2 \pi f_{m} t\right), m=0,1, \ldots, M-1,
$$

where $w_{m}$ is the amplitude weighting and $w_{e}\left(f_{m}\right)$ is the element pattern factor which can be expressed as [28]

$$
w_{e}\left(f_{m}\right)=\operatorname{sinc}\left(\frac{\pi a f_{m} \sin (\theta)}{c_{0}}\right),
$$

where $\theta$ is the direction angle, $c_{0}$ is the speed of light, and $\operatorname{sinc}(x)=\sin (x) / x$. In order to evaluate the impacts of the frequency offsets on the $w_{e}\left(f_{m}\right)$, we define the following performance metric:

$$
y=\frac{w_{e}\left(f_{m}\right)}{w_{e}\left(f_{0}\right)} .
$$

Suppose $\Delta f=5 \mathrm{kHz}$, Fig. 2 shows the ratio $\frac{w_{e}\left(f_{m}\right)}{w_{e}\left(f_{0}\right)}$ as a function of the number of array elements. It is noticed

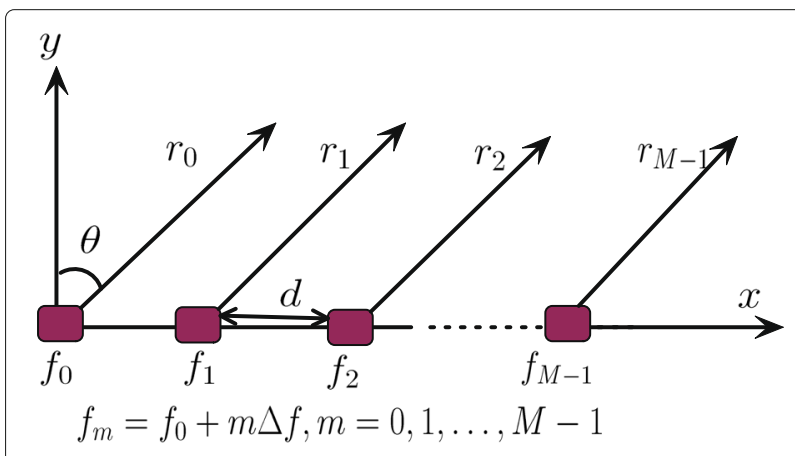

Fig. 1 Illustration of a linear FDA transmitter 


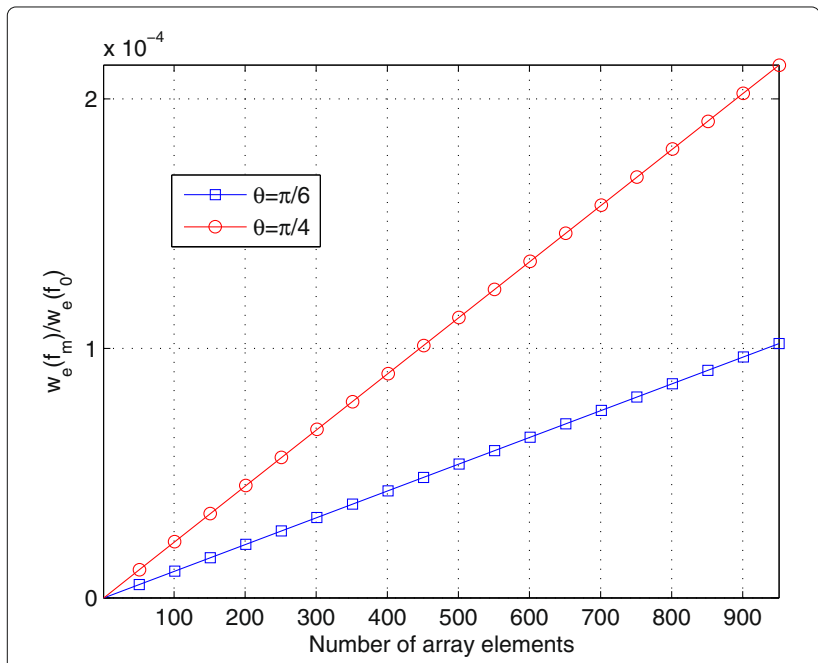

Fig. 2 The ratio $\frac{w_{e}\left(f_{m}\right)}{w_{e}\left(f_{0}\right)}$ versus the number of array elements

that the frequency offsets have ignorable effects on the amplitude changes of the $w_{e}\left(f_{m}\right)$, and thus, $w_{e}\left(f_{m}\right)=1$ is adopted in subsequent discussions.

Different from traditional phased-array antenna, which uses the same carrier frequency for all the array elements, the FDA uses different carrier frequencies with small frequency offsets for the array elements. This implies that the FDA elements will have frequency-dependent beam steering component. The radiation frequency $f_{m}$ is [4]

$$
f_{m}=f_{0}+m \Delta f \text {, }
$$

with $f_{0}$ and $M$ being the carrier frequency and number of array elements, respectively. The signal arriving at a farfield point with slant range $r$ for the first element and direction angle $\theta$ can be expressed as

$$
s_{m}\left(t-\frac{r_{m}}{c_{0}}\right) \doteq w_{m} w_{e}\left(f_{m}\right) \exp \left\{-j 2 \pi f_{m}\left(t-\frac{r_{m}}{c_{0}}\right)\right\}
$$

where $r_{m}$ can be approximated by

$$
r_{m} \approx r-m d \sin \theta,
$$

with $d$ being the element spacing.

To avoid aliasing effects, the element spacing should be smaller than the half wavelength of the highest frequency signal. Generally, positive frequency offset $\Delta f$ is assumed in most of literatures. Certainly, negative frequency offset $\Delta f$ is also feasible for the FDA radar. Therefore, in this paper the uniform array element spacing is designed as

$$
d=\frac{1}{2} \frac{c_{0}}{f_{0}+(M-1)|\Delta f|} .
$$

If the amplitude and beam steering components are all equal to one, namely, $w_{m}=1$ and $w_{e}\left(f_{m}\right)=1$, the array factor seen at the target position $(r, \theta)$ can be derived as [19]

$$
\begin{aligned}
\operatorname{AF}(t ; r, \theta) \doteq & \sum_{m=0}^{M-1} \exp \left\{-j 2 \pi f_{m}\left(t-\frac{r_{m}}{c_{0}}\right)\right\} \\
= & \exp \left\{j \Phi_{0}\right\} \sum_{m=0}^{M-1} \exp \left\{-j 2 \pi\left(m \Delta f t-m \frac{\Delta f r}{c_{0}}\right.\right. \\
& \left.\left.+m \frac{d f_{0} \sin \theta}{c_{0}}+m^{2} \frac{\Delta f d \sin \theta}{c_{0}}\right)\right\}
\end{aligned}
$$

where the common phase $\Phi_{0}$ is $\Phi_{0}=-2 \pi f_{0}\left(t-\frac{r}{c_{0}}\right)$.

To get a closed-form expression, we approximate the phase term $m^{2} \Delta f d \sin \theta / c_{0}$ as $m \Delta f d \sin \theta / c_{0}$. To make the assumption reasonable, an empirical requirement can be employed:

$$
\left|\frac{(M-1)^{2} \Delta f d \sin \theta}{c_{0}}-\frac{(M-1) \Delta f d \sin \theta}{c_{0}}\right| \leq \frac{\pi}{4} .
$$

Suppose the carrier frequency is $f_{0}=10 \mathrm{GHz}$, the azimuth angle is $\theta=\pi / 3$ and the element spacing is half of the maximal wavelength. Figure 3 shows the maximum allowable frequency increment as a function of the number of array elements, $M$. Since Eq. (9) is just an empirical phase requirement, the high offsets shown in Fig. 2 cannot be used in practice due to bandwidth constraints. In order to avoid also frequency decorrelation to happen in target response, in most literature the frequency offset $\Delta f$ is assumed to be smaller than one thousandth of the transmitted signal bandwidth $B_{r}$. It is observed that the assumption requirement is easily achieved for practical

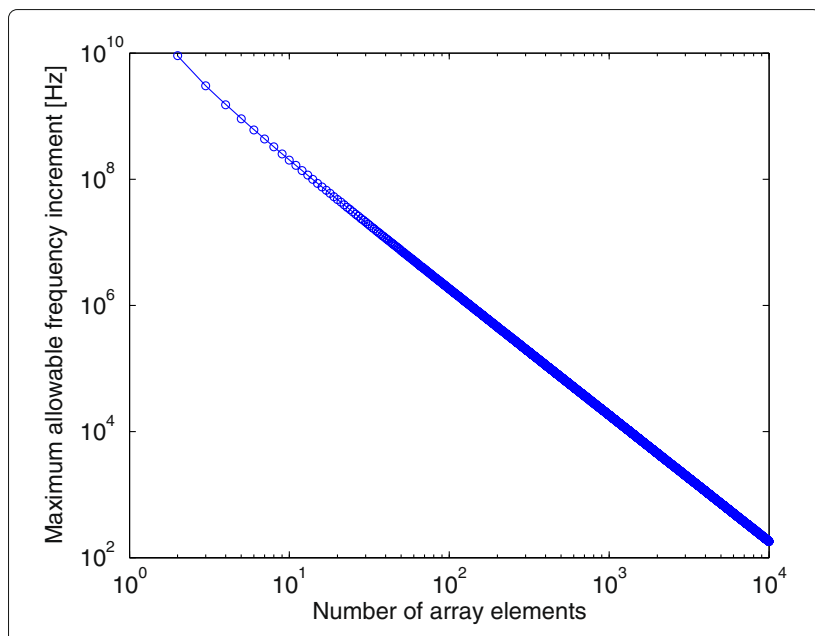

Fig. 3 Maximum allowable frequency offset versus the number of array elements 
FDA radar systems. In this case, Eq. (8) can be rewritten in a closed-form as

$$
\begin{aligned}
\operatorname{AF}(t ; r, \theta) \approx & \exp \left\{j \Phi_{1}\right\} \\
& \times \frac{\sin \left[M \pi\left(\Delta f t-\frac{\Delta f r}{c_{0}}+\frac{d f_{0} \sin \theta}{c_{0}}+\frac{\Delta f d \sin \theta}{c_{0}}\right)\right]}{\sin \left[\pi\left(\Delta f t-\frac{\Delta f r}{c_{0}}+\frac{d f_{0} \sin \theta}{c_{0}}+\frac{\Delta f d \sin \theta}{c_{0}}\right)\right]},
\end{aligned}
$$

where $\Phi_{1}$ is

$$
\Phi_{1}=\Phi_{0}+\pi(M-1)\left[\frac{\Delta f r}{c_{0}}-\frac{f_{0} d \sin \theta}{c_{0}}-\frac{\Delta f d \sin \theta}{c_{0}}\right] .
$$

According to Eq. (10), the transmit beampattern achieves the maximum at

$$
\Delta f t-\frac{\Delta f r}{c_{0}}-\frac{d}{\lambda} \sin \theta=k, k=0,1, \ldots
$$

When the time variable $t$ is fixed, the FDA beampattern will be coupled in the range and angle dimensions, caused by the synchronous linearly changing between frequency increment and element spacing. If this synchronization is damaged, the FDA may yield uncoupled range-angle beampattern. Hence, we have two potential solutions:

1. Linearly increasing frequency offsets and nonuniform linear array

2. Uniform linear array and non-linearly increasing frequency offsets

In this paper, we use Costas-sequence modulated frequency increments, namely, the second case.

\section{FDA radar using Costas-sequence modulated frequency offsets}

In the Costas frequency coding scheme, the columns represent $M$ contiguous time slices (each of duration $t_{b}$ ) and the rows represent $M$ distinct frequencies, equally spaced by $\Delta f_{0}$. We use this scheme for the FDA, namely, only one carrier frequency is transmitted by any one of he $M$ FDA elements and each carrier frequency is used only once. The construction algorithms for Costas signals were discussed by Golomb and Taylor [29]. The coding sequence, the order of used frequencies is a concise way to describe the coding matrix. With regard to the difference matrix, note that the top row and the leftmost column are headings and not part of the matrix. The element of the difference matrix in row $i$ and column $j$ is

$$
D_{i, j}=a_{i+j}-a_{j}, i+j \leq M,
$$

where $a_{i}$ is the $i$ th element of the coding sequence. The remaining locations (where $i+j>M$ ) are left blank. This equation implies that the first row is formed by taking differences between adjacent elements in the coding sequence, the second row is formed by taking differences between next-adjacent elements, and so on.

Construction of Costas codes can be understood as a construction of stepped-frequency waveforms, where the pulse width $\tau$ is divided into $M$ sub-pulses, each of width $\tau_{1}$. Within each group of $M$ sub-pulses, the frequency is increased by $\Delta f$ from one subpulse to the next. The total signal bandwidth is $(M-1) \Delta f$ and there are $M$ sub-pulses; each subpulse has the duration of $1 / \Delta f$, then the time-bandwidth product of the transmitted signal is $(M-1) \Delta f \times M \times 1 / \Delta f=(M-1) M$. Costas codes are similar to stepped-frequency waveforms, except that the frequencies for the subpulse are selected in a random fashion, according to some predetermined rule or logic. Figure 4 compares the hopping orders of LFM and Costas coding schemes, where the $x$-axis and $y$-axis denote the time and frequency, respectively.

The normalized complex envelope of the Costas signal can be expressed as [30]

$$
s(t)=\frac{1}{\sqrt{M \tau_{1}}} \sum_{m=0}^{M-1} u_{m}\left(t-m \tau_{1}\right),
$$

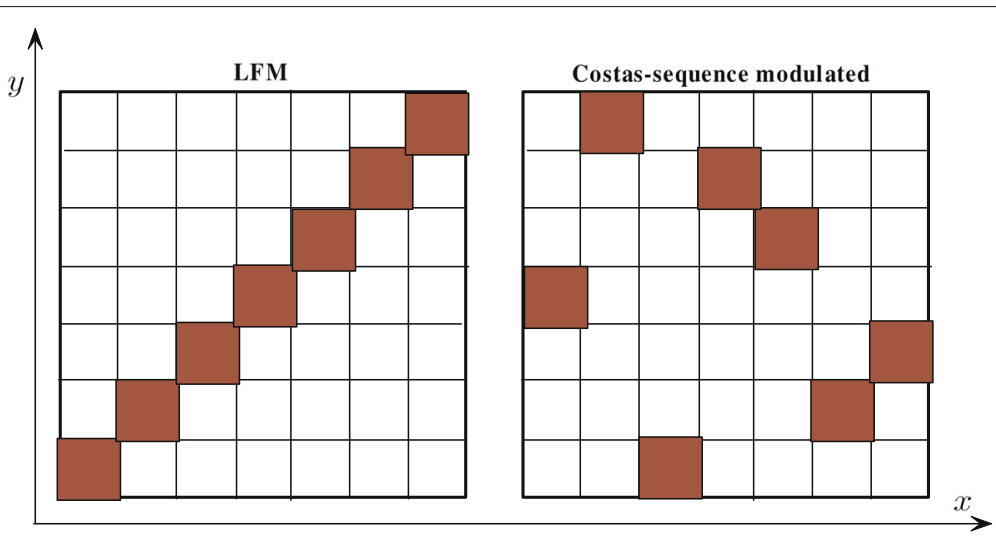

Fig. 4 Binary matrix representation of quantized LFM and Costas coding 
where $u_{m}(t)=\exp \left(j 2 \pi f_{m} t\right), 0 \leq t \leq \tau_{1}$. Note that the sub-pulses are separated in time-domain. It is easily understood that the hopping order strongly affects the ambiguity function of the signal. The ambiguity function can be predicted roughly by overlapping a copy of the binary matrix on itself and then shifting one relative to the other according to the desired delay and Doppler. The corresponding ambiguity function of the matched filter is

$$
\begin{aligned}
\chi(\tau, \nu)= & \frac{1}{M} \sum_{m=0}^{M-1} \exp (j 2 \pi m \nu \tau) \\
& \times\left\{\Phi_{m m}(\tau, v)+\sum_{q=0, q \neq m}^{M-1} \Phi_{m q}\left(\tau-(m-q) \tau_{1}, v\right)\right\},
\end{aligned}
$$

where

$$
\begin{aligned}
& \Phi_{m q}(\tau, \nu)=\left(\tau_{1}-\frac{|\tau|}{\tau_{1}}\right) \frac{\sin \alpha}{\alpha} \exp \left(-j \beta-j 2 \pi f_{q} \tau\right) \\
& \alpha=\pi\left(f_{m}-f_{q}-v\right)\left(\tau_{1}-|\tau|\right) \\
& \beta=\pi\left(f_{m}-f_{q}-v\right)\left(\tau_{1}+|\tau|\right) .
\end{aligned}
$$

As noted in Eq. (14), in the standard Costas sequence the sub-pulses corresponding to each element are not aligned. However, in the FDA the transmitted signals from all the elements should be aligned in time-domain; otherwise, the beampattern of the whole array will be decided mainly by some particular elements for a given instant. To avoid this problem, we use the Costas-sequence modulated frequency offsets in a time-aligned way. Taking the Costas sequence illustrated in Fig. 4 as an example, the adopted frequency indexes in the seven time intervals are $\{4,7,1,6,5,2,3\}$. Accordingly, our method allows for a seven-element FDA and the seven elements use the frequency offsets $4 \Delta f_{0}, 7 \Delta f_{0}, 1 \Delta f_{0}, 6 \Delta f_{0}, 5 \Delta f_{0}, 2 \Delta f_{0}$, and $3 \Delta f_{0}$ with $\Delta f_{0}$ being the hopping frequency step, respectively. That is, similar to conventional FDA, all the signals are transmitted simultaneously from the Costas modulated FDA.

The frequency fed to the $m$ th element of the FDA using Costas-sequence modulated frequency offsets can be generally written as

$$
f_{m}=f_{0}+\Delta f_{m}, m=0,1,2, \ldots, M-1,
$$

where $\Delta f_{m}$ is the frequency increment for the $m$ th element. The transmitted signal of the $m$ th element can then be expressed as

$$
s_{m}(t)=\exp \left(j 2 \pi\left(f_{0}+\Delta f_{m}\right) t\right) .
$$

For an ideal point target at the range $r$ and azimuth $\theta$, the received echo corresponding to the $m$ th antenna is

$$
r_{m}(t)=s_{m}\left(t-2 \frac{r+m d \sin \theta}{c_{0}}\right) .
$$

By demodulating the received returns with the transmit signal, we can get the baseband signal:

$$
b_{m}(r, \theta)=\exp \left\{-j \frac{4 \pi}{c_{0}}\left(f_{0}+\Delta f_{m}\right)(r+m d \sin \theta)\right\} .
$$

For notation convenience, the above equation can be simply rewritten as

$$
b_{m}(r, \theta)=\exp \left\{-j \phi_{m}\right\}
$$

where $\phi_{m}$ is

$$
\phi_{m}=\frac{4 \pi}{c_{0}}\left(f_{0}+\Delta f_{m}\right)(r+m d \sin \theta) .
$$

In the single snapshot case, the received noise-free echo of one ideal target can be represented as the following receive steering vector

$$
\mathbf{b}(r, \theta)=\left[\begin{array}{llll}
e^{-j \phi_{0}} & e^{-j \phi_{1}} \ldots e^{-j \phi_{m}} \ldots e^{-j \phi_{M-1}}
\end{array}\right]^{T},
$$

where ${ }^{T}$ is the transpose operator. For the multi-target case, the received echo vector for the $k$ th snapshot can then be expressed as

$$
\mathbf{x}(k)=\sum_{p=1}^{P} \alpha_{p}(k) \mathbf{b}\left(r_{p}, \theta_{p}\right)+\mathbf{n}(k), k=1,2, \ldots, K,
$$

where $\alpha_{p}(k), r_{p}$, and $\theta_{p}$ are the reflection coefficient, slant range, and azimuth angle for the $p$ th target at the $k$ th snapshot, respectively, $P$ is the target number, $K$ is the snapshot number, and $\mathbf{n}(k)$ is the $M \times 1$ additive receiver noise vector. Note that the target reflection coefficient $\alpha_{p}(k)$ may vary from shapshot to snapshot [31].

Adaptive beamforming algorithms can be used to optimally design the weighting vector $\mathbf{w}$ to synthesize the desired transmit-receive beampattern. Specifically, when the non-adaptive beamforming algorithm is adopted, the weighting vector is

$$
\mathbf{w}=\mathbf{b}\left(r_{0}, \theta_{0}\right) \text {, }
$$

where $\theta_{0}$ and $r_{0}$ denote the angle and range of the desired target, respectively. In this case, the maximum is steered to the expected location $\left(r_{0}, \theta_{0}\right)$. The FDA radar transmitreceive beampattern can then be expressed as

$$
G(r, \theta) \triangleq \frac{\left|\mathbf{b}^{H}\left(r_{0}, \theta_{0}\right) \mathbf{b}(r, \theta)\right|^{2}}{\left\|\mathbf{b}\left(\theta_{0}, r_{0}\right)\right\|^{4}} .
$$

It is noticed that, like a phased-array radar, the FDA radar has coherent transmit processing gain; however, the FDA radar directional gain depends on both the range and angle parameters, whereas the phased-array radar 
directional gain depends only on the range parameter. This range-angle-dependent beam provides a potential approach to suppress range-dependent interferences and noise.

The conventional non-adaptive beamforming is known to be optimal in the sense that it provides the highest possible output signal-to-noise ratio (SNR) and signal-tointerference plus noise ratio (SINR) in the background of white Gaussian noise [32]. The output SINR of the FDA radar can be evaluated by

$\mathrm{SINR} \bumpeq \frac{\sigma_{s}^{2} M^{2}}{\sum_{i} \sigma_{i}^{2}\left|\mathbf{b}^{H}\left(r_{0}, \theta_{0}\right) \mathbf{b}\left(r_{i}, \theta_{i}\right)\right|^{2}\left|\mathbf{b}^{H}\left(r_{0}, \theta_{0}\right) \mathbf{b}\left(r_{i}, \theta_{i}\right)\right|^{2}+\sigma_{n}^{2} M}$,

where $\sigma_{s}^{2}$ is the variance of the desired target signal, $\sigma_{i}^{2}$ is the variance of the $i$ th interference, and $\sigma_{n}^{2}$ is the noise variance. If the target is observed in the background of few weak interferences which are well separated from the target, the interference-to-noise power can be attributed to the noise term only. In this case, the SINR for the FDA radar simplifies to

$$
\operatorname{SINR} \simeq \frac{\sigma_{s}^{2} M}{\sigma_{n}^{2}},
$$

which means that the FDA radar has an equivalent robustness again noise.

In contrast, if the target is observed in the background of strong interferences, then we can fairly consider the noise power to be negligible as compared to the interference power. In such a case, we have

$$
\mathrm{SINR} \simeq \frac{\sigma_{s}^{2} M^{2}}{\sum_{i} \sigma_{i}^{2}\left|\mathbf{b}^{H}\left(r_{0}, \theta_{0}\right) \mathbf{b}\left(r_{i}, \theta_{i}\right)\right|^{2}\left|\mathbf{b}^{H}\left(r_{0}, \theta_{0}\right) \mathbf{b}\left(r_{i}, \theta_{i}\right)\right|^{2}} .
$$

The FDA using Costas-sequence modulated frequency offsets will make the transmit-receive beampattern mainlobe approximate an ideal thumbtack response, as provided in the next section. In this case, the SINR (Eq. (31)) can be simplified as

$$
\operatorname{SINR} \simeq \frac{\sigma_{s}^{2} M^{2}}{\bar{\sigma}_{i}^{2}},
$$

where $\bar{\sigma}_{i}^{2}$ denotes the mean of $\sigma_{i}^{2}$. Thus, it is expected that this FDA radar has better robustness against interferences than both conventional phased-array radar and standard FDA radar.

\section{Numerical results}

It is well known that when a given delay-Doppler shift results in a coincidence of $N$ points, the ambiguity function is expected to yield a peak of approximately $N / M$ at the corresponding delay-Doppler coordinate. For the linearly frequency modulated (LFM) coding case, only delay and Doppler shifts of equal number of units, namely, $\tau=$ $m t_{b}, v=m \Delta f, m=0, \pm 1, \ldots, \pm(M-1)$, will cause an overlap of dots, and the number of coinciding dots will be $N=M-|m|$. This hints at a diagonal ridge in the ambiguity function, along the line $v=\Delta f \tau / t_{b}$. What is unique for a Costas signal is that the number of coinciding dots cannot be larger than one for all but the zero-shift case, where all dots coincide $(N=M)$. This property implies a narrow peak of the ambiguity function at the origin and low sidelobes elsewhere. If $\Delta f=1 / \tau_{1}$, the exact ambiguity function values at the grid points will be either 1 or 0 , according to the corresponding number of coinciding dots.

As an example, we consider the Welch-Constructed Costas coding sequence for $M=12$, namely, the chosen positions are $1,2,4,8,3,6,12,11,9,5,10$, and 7 . This implies that the frequency offsets are not linearly increasing as the element index, but change as the Costas sequence, namely, $\left\{1 \Delta f_{0}, 2 \Delta f_{0}, 4 \Delta f_{0}, 8 \Delta f_{0}, 3 \Delta f_{0}, 6 \Delta f_{0}\right.$, $\left.12 \Delta f_{0}, 11 \Delta f_{0}, 9 \Delta f_{0}, 5 \Delta f_{0}, 10 \Delta f_{0}, 7 \Delta f_{0}\right\}$. Suppose $\Delta f_{0}=$ $100 \mathrm{kHz}$, Fig. 5a shows the ambiguity function for a monochromatic waveform with Costas-sequence modulated frequency offsets for the FDA radar. As a comparison, suppose the following parameters for the LFM waveform: bandwidth is $10 \mathrm{MHz}$, starting frequency is $0 \mathrm{~Hz}$ and pulse duration is $1 \mu s$. Figure $5 \mathrm{~b}, \mathrm{c}$ show the ambiguity function for a LFM waveform with Costas-sequence modulated frequency offsets for FDA and phased-array radars, respectively. It is noticed that the use of Costas-sequence modulated frequency offsets produces a more focused peak. The ambiguity function sidelobes are usually lower than $20 \log _{10}(2 / M)$, but the near sidelobes are higher decaying in a manner typical of the sidelobes of a signal with a rectangular spectrum.

Next, we consider the transmit beampattern for the arrays. Suppose $\Delta f=5 \mathrm{kHz}$ and use also the WelchConstructed Costas sequence with $M=12$, Fig. 6 a shows the Costas-sequence modulated FDA using uniform transmit weighting coefficients. It is seen that the transmit beampattern has random-like peak distribution without obvious peaks. This implies that it is difficult to be detected or localized by an unfriendly detector without knowing the specified coding sequence for the frequency increments, and it can be exploited to develop low probability interception (LPI) or radio frequency (RF) stealth radar techniques [33]. However, with the knowledge of the specific Costas sequences used in the FDA transmitter, we can recover the thumbtack transmit-receive beampattern for the targets. As a comparison, Fig. 6b provides the standard FDA using also uniform transmit weighting coefficients, which produces range-angle-dependent and range-angle coupling transmit beampattern. This implies 

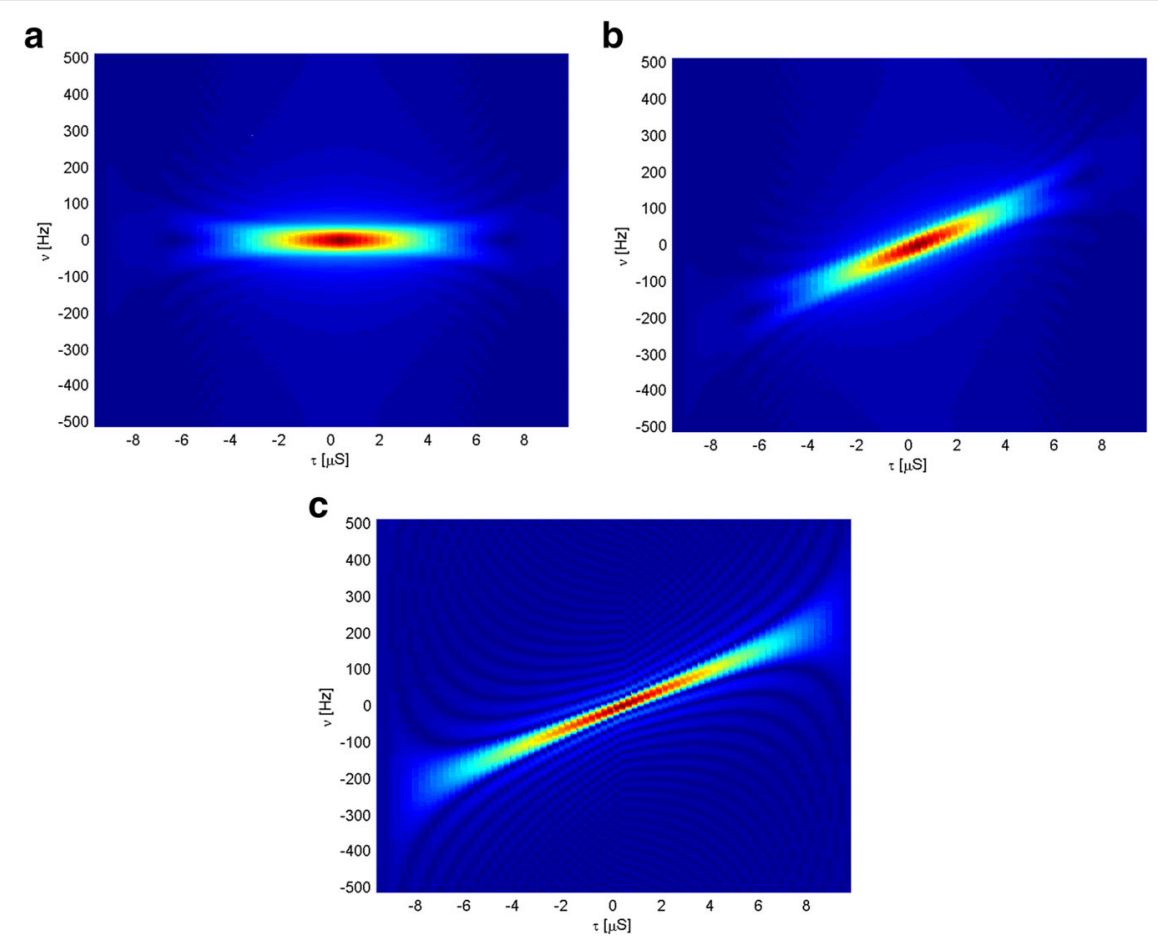

Fig. 5 Comparisons of ambiguity functions. a Monochromatic waveform with Costas-sequence modulated frequency offset for FDA. b LFM waveform with Costas-sequence modulated frequency offset for FDA. c LFM waveform without Costas-sequence modulated frequency offset for phased-array
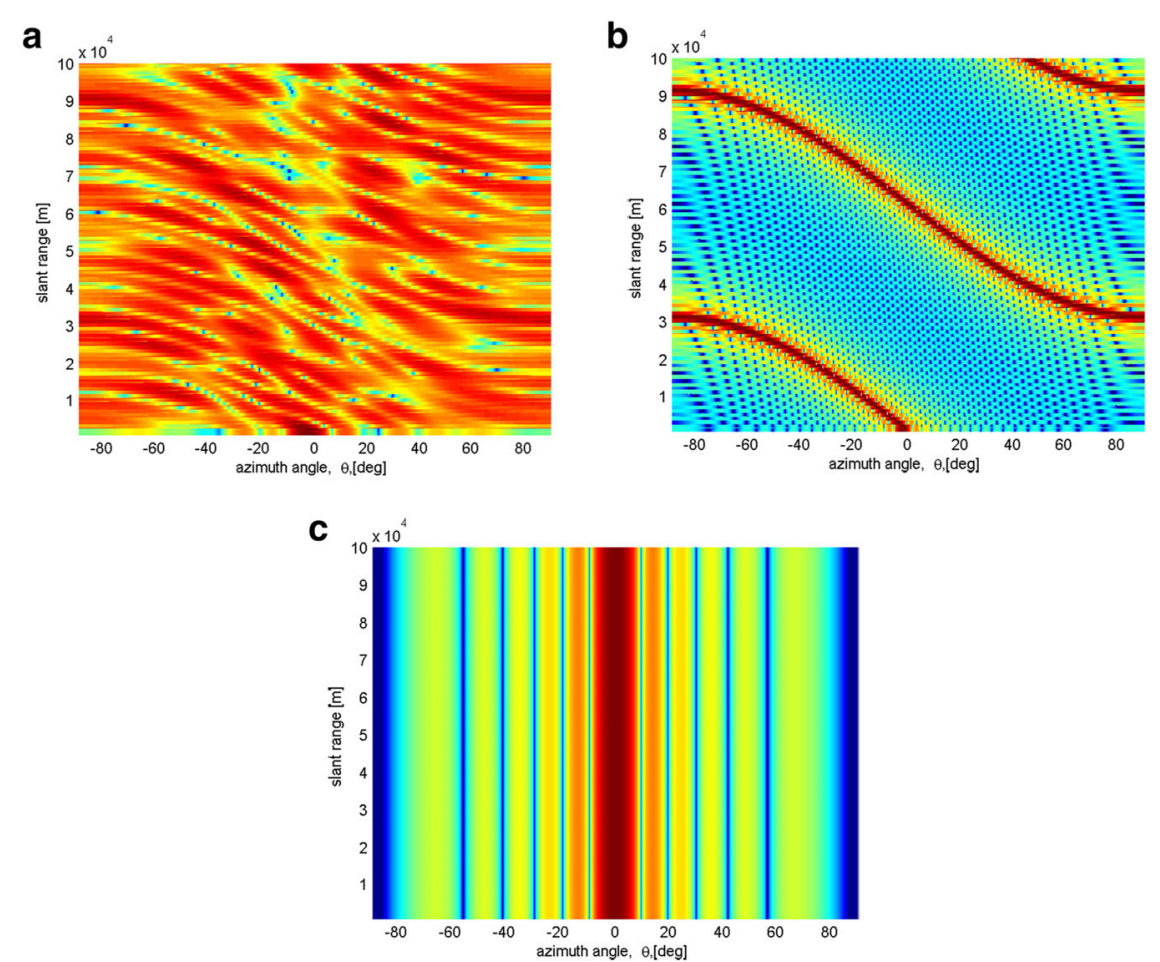

Fig. 6 Comparisons of transmit beampatterns with uniform weighting coefficients. a Costas-sequence modulated FDA. b Standard FDA. c Traditional phased-array 

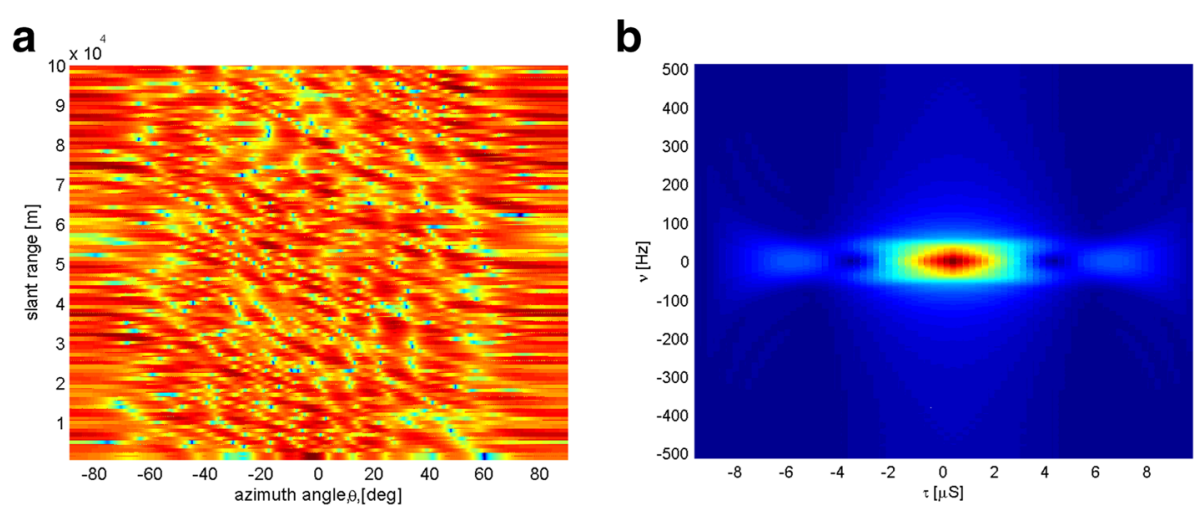

Fig. 7 Transmit beampattern and radar ambiguity function of the FDA radar using the Costas sequence with $M=18$. a Transmit beam-pattern. b Radar ambiguity function

that it can be exploited for range-angle localization of targets, but it may produce ambiguous results due to the range-angle coupling beampattern. Differently, the traditional phased-array generate angle-dependent only transmit beampattern, as shown in Fig. 6c, but it provides no range information of the targets because of its range-independent beampattern [34].

Finally, we consider another example to compare the differences between different length of Costas sequences. Using the Welch-Constructed Costas coding sequence with $M=18$, namely, the chosen positions are $1,2,4,8,16,13,7,14,9,18,17,15,11,3,6,12,5$, and 10 . Figure 7 shows the corresponding transmit beampattern and radar ambiguity function for $\Delta f_{0}=100 \mathrm{kHz}$. The results validate again that the FDA using Costas-sequence modulated frequency offsets produces random-like peak distribution without obvious peaks, and consequently, it is difficult to be detected by unfriendly detectors. Nevertheless, it generates also a focused peak in the radar ambiguity function. Therefore, we can conclude that LPI can be achieved by the FDA radar using Costas-sequence modulated frequency offsets.

\section{Conclusions}

This paper proposed a ULA FDA using Costas sequence modulated frequency offsets to decouple the range-angledependent beampattern by producing random-like energy distribution in the transmit beampattern. Moreover, a thumbtack transmit-receive beampattern can be obtained at the receiver. In doing so, the range and angle of targets can be solely estimated through matched filtering and subspace decomposition algorithms in the receiver signal processor. Numerical results show that the proposed scheme outperforms the standard FDA in focusing the transmit energy, especially in the range dimension. This Costas-sequence modulated FDA radar can be exploited for LPI radar applications due to the random transmit beampattern and thumbtack transmit-receive beampattern. Such a topic is planned for our future work.

\section{Acknowledgements}

This work was supported by the National Natural Science Foundation of China under grant 61571081, Sichuan Province Science Fund for Distinguished Young Scholars under grant 2013JQ0003, and Sichuan Technology Research and Development fund under grant 2015GZ0211.

\section{Competing interests}

The authors declare that they have no competing interests.

Received: 20 June 2016 Accepted: 5 November 2016

Published online: 23 November 2016

\section{References}

1. PF McManamon, PJ Bos, MJ Escuti, J Heikenfeld, S Serati, H Xie, EA Watson, A review of phased array steering for narrow-band electrooptical systems. Proc. IEEE. 97(6), 1078-1096 (2009)

2. J Li, P Stoica, The phased array is the maximum SNR active array. IEEE Signal Process. Mag. 27(2), 143-144 (2010)

3. SD Greve, P Ries, FD Lapierre, JG Verly, Framework and taxonomy for radar space-time adaptive processing (STAP) method. IEEE Trans. Aerosp. Electron. Syst. 43(3), 1084-1099 (2007)

4. P Antonik, MC Wicks, HD Griffiths, CJ Baker, in Proc. IEEE Radar Conference. Frequency diverse array radars (IEEE, New Jersey, 2006), pp. 215-217

5. M Secmen, S Demir, A Hizal, T Eker, in Proc. IEEE Radar Conference. Frequency diverse array antenna with periodic time modulated pattern in range and angle (IEEE, New Jersey, 2007), pp. 427-430

6. S Huang, KF Tong, CJ Baker, in Proc. IEEE Antennas and Propagation Conference. Frequency diverse array with beam scanning feature (IEEE, New Jersey, 2008), pp. 1-4

7. P Antonik, An investigation of a frequency diverse array. PhD thesis. (University College London, 2009)

8. TX Zhang, X-G Xia, LJ Kong, IRCI free range reconstruction for SAR imaging with arbitrary length OFDM pulse. IEEE Trans. Signal Process. 62(18), 4748-4759 (2014)

9. S Gogineni, A Nehorai, Frequency-hopping code design for MIMO radar estimation using sparse modeling. IEEE Trans. Signal Process. 60(6), 3022-3035 (2012)

10. GL Cui, HB Li, M Rangaswamy, MIMO radar waveform design with constant modulus and similarity constraints. IEEE Trans. Signal Process. 62(2), 343-353 (2014)

11. C Vazquez, C Garcia, Y Alvarez, S Ver-Hoeye, F Las-Heras, Near-field characterization of an imaging system based on a frequency scanning antenna array. IEEE Trans. Antennas Propag. 61(5), 2874-2879 (2013)

12. W-Q Wang, HC So, HZ Shao, Nonuniform frequency diverse array for range-angle imaging of targets. IEEE Sensors J. 14(8), 2469-2476 (2014) 
13. K-J Koh, H Elyas, Time-interleaved phased arrays with parallel signal processing in RF modulation. IEEE Trans. Antennas Propag. 62(2), 677-689 (2014)

14. S Mustafa, D Simsek, HAE Taylan, in Proc. IEEE Radar Conference. Frequency diverse array antenna with periodic time modulated pattern in range and angle (IEEE, New Jersey, 2007), pp. 427-430

15. T Higgins, S Blunt, in Proceedings of the 4th International Waveform Diversity \& Design Conference. Analysis of range-angle coupled beamforming with frequency diverse chirps (IEEE, New Jersey, 2009), pp. 140-144

16. L Z huang, XZ Liu, in Proceedings of the International Radar Conference. Precisely beam steering for frequency diverse arrays based on frequency offset selection (IEEE, New Jersey, 2009), pp. 1-4

17. W-Q Wang, HZ Shao, JY Cai, Range-angle-dependent beamforming by frequency diverse array antenna. Int. J. Antennas Propag. 2012, 1-10 (2012)

18. W-Q Wang, Overview of frequency diverse array in radar and navigation applications. IET Radar Sonar Navig. 10(6), 1001-1012 (2016)

19. PF Sammartino, CJ Baker, HD Griffiths, Frequency diverse MIMO techniques for radar. IEEE Trans. Aerosp. Electron. Syst. 49(1), 201-222 (2013)

20. W-Q Wang, HC So, Transmit subaperturing for range and angle estimation in frequency diverse array radar. IEEE Trans. Signal Process. 62(8), 2000-2011 (2014)

21. J Xu, GS Liao, SQ Zhu, L Huang, HC So, Joint range and angle estimation using MIMO radar with frequency diverse array. IEEE Trans. Signal Process. 63(13), 3396-3410 (2015)

22. W-Q Wang, HZ Shao, Range-angle localization of targets by a double-pulse frequency diverse array radar. IEEE J. Selected Topics Signal Process. 8(1), 106-114 (2014)

23. W-Q Wang, Subarray-based frequency diverse array radar for target range-angle estimation. IEEE Trans. Aerosp. Electron. Syst. 50(4), 3057-1076 (2014)

24. W Khan, IM Qureshi, S Saeed, Frequency diverse array radar with logarithmically increasing frequency offset. IEEE Antennas Wireless Propag. Lett. 14(1), 499-502 (2015)

25. W Khan, IM Qureshi, Frequency diverse array radar with time-dependent frequency offset. IEEE Antennas Wireless Propag. Lett. 13(1), 758-761 (2014)

26. HZ Shao, J Dai, J Xiong, H Chen, W-Q Wang, Dot-shaped range-angle beampattern synthesis for frequency diverse array. IEEE Antennas Wireless Propag. Lett. 15(1) (2016). in press

27. YM Liu, in Proc. IEEE International Conference on Acoustics, Speech, and Signal Processing. Range azimuth indication using a random frequency diverse array (IEEE, New Jersey, 2016), pp. 3111-3115

28. D Lynch, Introduction to RF Stealth. (SciTech Publishing, Raleigh, 2013)

29. SW Golomb, H Taylor, Constructions and properties of costas arrays. Proc. IEEE. 72(9), 1143-1163 (1984)

30. BR Mahafza, Z Elsherbeni, MATLAB Simulations for Radar Systems Design. (CRC Press, New York, 2003)

31. M Skolnik, Introduction to Radar Systems. (McGrow-Hill, New York, 2001)

32. HL Van Trees, Optimum Array Processing. (Wiley, New York, 2002)

33. W-Q Wang, Moving-target tracking by adaptive RF stealth radar using frequency diverse array antenna. IEEE Trans. Geosci. Remote Sens. 54(7), 3764-3773 (2016)

34. W-Q Wang, CL Zhu, Nested array receiver with time-delayers for joint target range and angle estimation. IET Radar Sonar Navig. 10 (2016). in press

\section{Submit your manuscript to a SpringerOpen ${ }^{\circ}$ journal and benefit from:}

- Convenient online submission

Rigorous peer review

- Immediate publication on acceptance

- Open access: articles freely available online

- High visibility within the field

- Retaining the copyright to your article

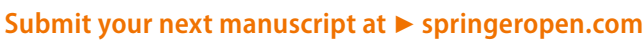

